\title{
A Survey of Various Ant Colony Optimization based Routing Protocols for Mobile Ad hoc Networks
}

\author{
Kanishka Raheja \\ Asst. Prof \\ Department of Comp. Sci. \& Eng \\ SGT University, Gurugram, India
}

\author{
Reenu Batra \\ Asst. Prof \\ Department of Comp. Sci. \& Eng \\ SGT University, Gurugram, India
}

\author{
Manoj Kapil \\ Professor \\ Department of Comp.Sci.\& Eng. \\ SGT University, Gurugram, India
}

\begin{abstract}
Routing in MANET (Mobile Ad hoc Network) is a challenging task because of the mobile nature of the nodes in a network and topology changes very often and developing effective routing protocols for MANET is also a highly challenging task. To fulfil the multiple routing requirements as low control overhead, low packet delay, high packet delivery rate and adapting effectively to network topology changes and so on, are the issues which are emerging. Amidst lots of problems which are found to be NP-hard in routing, new ways to find approximate solutions have to be investigated. A lot of attention was attracted by Swarm intelligence inspired algorithms which are based on the Ant Colony Optimization meta heuristic technique because they can offer optimized solutions ensuring low control overhead, robustness etc. and presents framework for approximating solutions to NP-hard problems. This paper includes 1) Introducing the ACO technique and its principles 2) Various ACO based routing protocols 3 ) summary and conclusion.
\end{abstract}

\section{Keywords}

Aco, Manet, Si, Fant, Bant

\section{INTRODUCTION}

\subsection{Manet}

Mobile Ad hoc Network also known as Wireless Ad hoc Network or ad hoc wireless Network is an infrastructure less network of mobile devices connected wirelessly and is a continuously self configuring network. Each node can act as router, transmitter and receiver and forward the traffic that is unrelated to its use. Each device in a MANET changes its links frequently and is free to move around in any direction. At one time the node is in the range and another time it is not [1]. Therefore routing has become a highly challenging and central issue because of lack of centralised control. To overcome this issue and provide high packet delivery rate, low packet delay and low control overhead, many metaheuristics techniques have been introduced which are inspired by the biological systems in the past two decades [2].

Swarm Intelligence approach was introduced by Beni and Wang in 1993.SI is a computational Intelligence and is the collective behaviour of decentralised, self organised systems, natural or artificial [4]. A typical SI field comprising of group of simple agents which interact locally with each other and with the environment surrounding them [5].These type of interactions generate enormous benefits and lead to good behaviour. It takes good advantage of swarm therefore it is able to provide optimal solutions: Particle swarm optimization (PSO) and Ant Colony Optimization (ACO). According to the previous work which has been done, we have studied about the Ant colony optimization techniques and various research applied on them. In this paper we give an overview of various routing protocols based on this technique. The rest of the paper is organised as follows. In section 1.2 the history of Ants and its techniques are presented, then in section

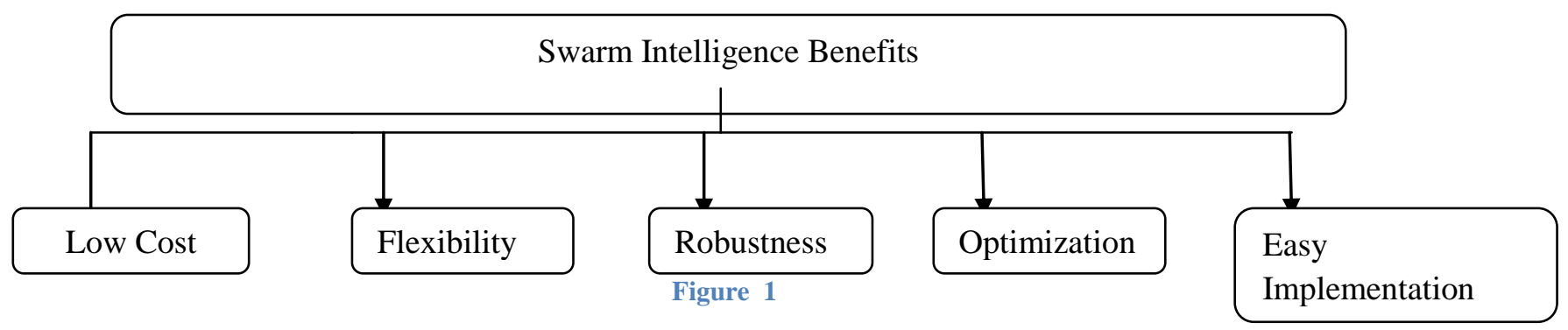

2 we have introduced the ACO based routing protocols. The comparison and summarization of the protocols reviewed in this paper and conclusion of the work is also mentioned.

\subsection{History of ACO}

Ants are the insects which are present everywhere and began to diversify 100 million years ago. Ants are very common type of insects and the size of ant colony can vary in its size up to millions even. An ant colony comprises of various castes such as workers, Queens, Drones and soldiers. Among them the most common category of Ants are Workers which are sterile females and do all of the work in the colony: searching for food, maintaining and expanding the nest, taking care of queen and brood, and so on. Queens are the fertile females which make all colonies, the main task of which is to lay eggs. The only male ants in the colony are the drones which only survive during the mating season. Soldiers are the other castes which can also be present in the colonies which protects all others from predators. Each caste in the colony has different tasks. Each and every caste in the colony has different tasks, all castes work together to ensure the survival of colony [9] [10]. To finish the task efficiently and remarkably single ant is not very good but a collection of ants called as colony can 
finish the task remarkably like as dealing with floods. To build raft in short time in order to beat the flood by using their bodies. Millions of ants can rearrange themselves to build a stable raft within some seconds, because they can react to their environment quickly.

The process of exchanging or sharing information among ants in an ant colony is different from that in humans, as humans use sound or sight to exchange information while ants use a chemical substance, pheromone which is the important component in communication. Whenever moving around ants produce pheromone through their glands and that is detected by the antennas in the surrounding area. Each ant in an ant colony understands different types of pheromone perfumes. There are various types of pheromone like alarm and trail pheromone. In order to alarm nest mates some species of ants create alarm pheromones by using poison glands. By detecting the alarm pheromone workers ants either escape or go fast or go towards danger: because of the presence of Formic acid and n-undecane. Second type of pheromone is the trail pheromone. When an ant foraging for food, it leaves its nest and randomly chooses a path to move on, if it does not finds a pheromone trail. Once it finds one, the highest probability is to choose that path. Whether it follows the route or not but it deposits pheromone over it. The same concept is used by other ants as well. Performance of a trail is directly related with the probability of an ant to follow that trail. When the food is exhausted and no more pheromone is deposited on the trail and pheromone begins to evaporate over the time [11].In this way ants are used to find the optimized solution.

\subsection{Artificial Ants}

As human Society follow the functions, ants take on similar functions, but ants don't rely on any central control to provide these functions. In previous work which is defined in [13] author designed a brilliant experiment: the double bridge experiment. Ant nest and food source are connected by two bridges of different length. In this experiment ants need to cover the route for foraging food. Experimental results showed that short path attracts more ants to follow, if both short and long path are given to the ants in the same time. Ant Colony Optimization Algorithm (ACO) was initially proposed by Dorigo in [7], the first Ant inspired algorithm, which is aimed to find the optimal path in a graph based on the foraging behaviour of Ants. Since after that various researches in this area was proposed by many scientists and many versions of ACO algorithms were proposed. ACO is the important components in the SI field and we will introduce more about this in the following sections.

\section{ACO BASED ROUTING PROTOCOLS}

$\mathrm{ACO}$ is a meta heuristic technique which is inspired by foraging behaviour of ants and is an important component of SI field. Artificial ants work together to find good solution for optimized problems [14]. If we recall the previous section, an ant deposits pheromone on its path to mark its trail and inform other ants. It becomes high probability to follow that trail for other ants and when they follow that route they deposit new pheromone over that route. That pheromone might attract new ants to follow and therefore it is an indirect information exchange among the ants. The problem under consideration which has to be optimized is to be represented graphically

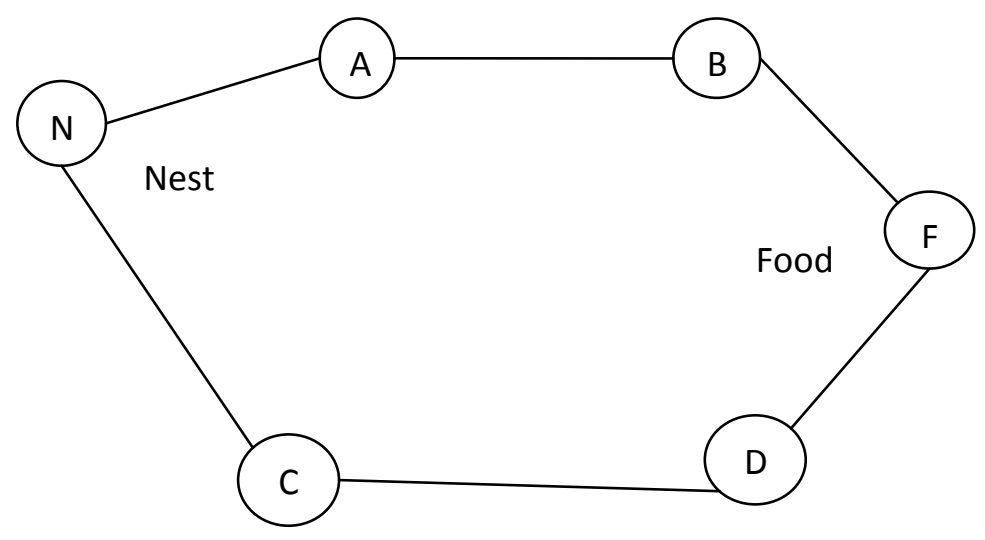

Figure. 2 Representation of nodes graphically while applying ACO metaheuristic

From graph it is needed to find the optimal path between the nodes $\mathrm{N} \& \mathrm{~F}$ as given in Fig 2. Once we formulate the problem on the graph the constraints of the problem need to be defined and the constraint is that ants can only move only on the arcs which connect the nodes.

A heuristic value and pheromone trail both are associated with an arc. Only one heuristic value is associated with an arc but the pheromone trail is separate for each destination [11]. If we consider the graph in fig. 2 the problem is to find the optimal path between the node $\mathrm{N}$ and F. We can say that the problem is to find the optimal solution between the ants' nest and food source. To determine the best route among the nodes and hence routing tables are continuously maintained by protocols.

As already discussed the behaviour of ant colonies is their foraging behaviour as when they visit each node some amount $\Delta \mathrm{t}$ of special substance called pheromone, they deposit.

$$
\Delta \mathrm{t} \alpha 1 / \mathrm{L}^{\Delta}(\mathrm{t}) \quad \text { II. } 1
$$

In the above equation $\mathrm{L}^{\Delta}(\mathrm{t})$ amount of pheromone deposited is directly proportional to the quality of route and 
quality of route is inversely proportional to the route length. This means if shortest path is followed by the ants then more is the amount of pheromone deposited on the route, that helps other ants to follow. In ant Colony Optimization, there is a mechanism to follow the old routes by evaporating the pheromone deposited on the route [15].This is necessary to eliminate old routes.

As arcs have the pheromone values and these pheromone values are updated by the ants that have completed the path length as follows:

$$
\mathrm{T}_{\mathrm{ij}}=(1-\Delta) \mathrm{xT}_{\mathrm{ij}}+{ }_{-}^{\mathrm{m}} \mathrm{m}_{-1 \mathrm{j}} \quad \text { II. } 2
$$

In the above equat: $K=1 \quad$ e number of ants that have completed the path, $\Delta \epsilon^{\prime}(0,1)$ determines the evaporation rate of pheromone. $\mathrm{T}_{\mathrm{ij}}{ }^{\mathrm{k}}$ is the quantity of pheromone deposited by ant $k$ on edge $(i, j)$.

ACO follows a probability rule to which is a function of pheromone trails and heuristic value. The probability rule can be represented as equation II.3 and II.4:

$$
P_{i j}(t)=\left\{\begin{array}{l}
\frac{\left[T_{i j}(t)\right]^{\alpha} \cdot\left[n_{i j}\right]_{\beta}}{\sum_{1 \in N i}\left[T_{i l(t)]}\right]^{\alpha} \cdot\left[n_{i j}\right]^{\beta}} \text { if } \mathrm{jEN}_{\mathrm{i}} \quad \text { II.3 } \\
\\
0 \text { if } \mathrm{jEN}_{\mathrm{i}} \text { II.4 }
\end{array}\right.
$$

In the above equation $\mathrm{P}_{\mathrm{ij}}$ denotes the probability of an ant to move from node $i$ to node $j$ at the $i^{\text {th }}$ time slot and $\mathrm{N}_{\mathrm{i}}$ is the set of current neighbouring nodes of node $i, T_{i j} i(t)$ the amount of pheromone deposited on arc between node $i$ and node $j$ at $i_{\text {th }}$ iteration step or time slot, $n_{\mathrm{ij}}$ is the heuristic value of the arc between node $i$ and node $j, \alpha$ and $\beta$ re weight parameters which maintains the pheromone intensity from node $i$ to node $\mathrm{j}$ [11].

Inspired by the ACO Algorithms, researchers have been motivated to apply these algorithms to find optimized routes for network communications .Now we introduce some of the famous approaches:

\subsection{Ant Net}

Ant Net was proposed by Di Caro and Dorigo [16] which is the first representative ACO Based Algorithm or solving the problem of routing in MANET. In this the concept of forward Ant and Backward Ant is used. Each node proactively in a network sends out forward Ants (FANT) to discover a path to randomly chosen destination node. Backward ants are sent back to the source node following the reverse path, whenever the forward ants reach the destination. Local routing tables and network status are updated by BANT at each node. The performance of this is evaluated in three different network scenarios.

\subsection{Ara}

This ACO based routing protocol was proposed by Gunes et al[17].This is based on simple ant colony meta heuristic algorithm and is an on demand routing protocol. This algorithm consists of three phases: a route discovery phase, route maintenance and route failure handling. In the route discovery phase, new routes are created. The creation of new routes require the use of forward ant and backward ant much similar like Ant Net algorithm. Sender broadcast the FANT. FANT is a small packet with unique number. Nodes are able to distinguish duplicate packets on the basis of sequence number and are deleted by intermediate nodes. Once FANT reach the destination BANT are created and sent back to the source node. If a node recognizes failure it first sends the pheromone value to zero so that that link may not be active. An alternate link will be searched after it. If this route also fails, then it informs its neighbours. This process is repeated until an alternative route has been found.

\subsection{Pera}

PERA was proposed by Baras and Mehta [18], which is proactive routing protocol. To discover the network topology PERA also uses ant like agents and maintain routing in dynamic networks as MANET. PERA uses three kinds of ants: regular FANT, uniform FANT and BANT. The ants which are sent proactively are regular and uniform FANT. These ants explore available route in a network. The routing procedure of Regular and uniform ants are different. Instead of using routing table at each node uniform FANTs chose the next hope node with equal probability. BANT are used to adjust the routing tables and statistic tables at each node, according to the information gathered by FANT. Comparison was done with AODV [19], a proactive routing protocol, results showed that PERA has better output in all parameters.

\subsection{AntHocNet}

This algorithm was developed by Di Caro et al [19] which is a hybrid multipath routing algorithm. Likewise the previous algorithms had FANT and BANT. This algorithm has 6 different kinds of ants: proactive FANT and proactive BANT, Reactive FANT and Reactive BANT, Repair FANT and Repair BANT. If a source node has no information which route is to be followed for the destination node, it broadcast the RFANT otherwise it unicasts. When this RFANT reaches the destination as the other algorithms do a RBANT is sent back to the source. When it travels back it collects all information about the links and updates the pheromone values at each node. AntHocNet starts the proactive route maintenance process once the first route is established. Then the PFANT are sent out to the destination node by the source node. When PFANT reaches the destination node it sends PBANT back to the source and update pheromone table at each link. When any link fails RPFANT and RPBANT are used o handle the problem. Author compared the results with AODV and find the results quite better than AODV.

\subsection{Paconet}

Osagie et al.[21] proposed PACONET a ACO algorithm with some enhancement in this. Instead of broadcasting the FANT to every node PACONET restricts the broadcasting to explore the network. When FANT visits the node it update the pheromone value and keep the record of all visited nodes and total time it has travelled. In a similar way like in the other algorithms has been described when FANT reach the destination, a corresponding BANT is generated and visit the nodes which were recorded by FANT and in the reverse direction again the pheromone value will be updated by BANT. Different from the other algorithms, this algorithm let both FANT and BANT to update the pheromone value. Result of this protocol was compared with AODV [2][11]. 


\subsection{Aco-Ahr}

This algorithm which is a hybrid routing algorithm includes reactive routing setup and proactive routing maintenance and was proposed by $\mathrm{Yu}$ et al. This algorithm uses two types of agents: Service agents and Ant agents. Ant agents comprises of FANT and BANT as in other algorithms. As described in the previous algorithm in the reactive route set up FANT records all the nodes it has visited along the route and each BANT carries all information recorded by FANT and it calculates the delay from one intermediate node to destination. Service agent is generated once a BANT reaches the source node and it updates the routing tables at intermediate nodes by information which is gathered by BANT.As the route maintenance is proactive proactive FANT are sent out while the data session is going.

\subsection{Hopnet}

HOPNET, which is proposed by WANG et al [22] and is based on the zone routing framework with ACO algorithm HOPNET performs local proactive route discovery within the node's neighbourhood and reactive communication between neighbourhoods. Authors have compared the result of HOPNET with famous routing protocols such as AODV, AntHocNet etc. Results showed that HOPNET is highly scalable in large networks.

\subsection{Summary and conclusion:}

In this paper we reviewed the Ant Colony Optimization algorithm and various routing protocols which were proposed in the early stages of ACO based routing protocols. First ACO based protocol was AntNet, proposed in 1998 gave example of how to apply the algorithm in communication networks. In the following 10 years many researchers proposed many ACO protocols for MANET. The main aim was to find out the optimal path from source to destination node. After reviewing it was concluded that using ACO algorithm on MANET protocols lead to good results in overhead, end to end delay, PDR etc.

\section{REFERENCES}

[1] K. A. Gupta, Harsh Sadawarti, K. A. Verma.2010 Performance analysis of AODV, DSR and TORA Routing Protocols. International Journal of Engineering and technology (IJET), ISSN: 1793-8236, Article No. 125, Vol.2 No. 2, April, pp. -Yaode

[2] F. Dressler and O. B. Akan.2010 .A survey on bioinspired networking. Comput. Netw., vol. 54, no. 6, pp. 881_900

[3] G. Beni and J. Wang.1993. Swarm intelligence in cellular robotic systems. In Robots Biological Systems: Towards a New Bionics?. Berlin, Germany: Springer, $\quad$ pp. 703_712, doi: https://doi.org/10.1007/978-3-642-58069-7_38.

[4] K. Kordon.2010.Swarm intelligence: The benefits of swarms. In Applying Computational Intelligence. Berlin, Germany: Springer, pp. 145_174, doi: https://doi.org/10.1007/978-3-540-69913-2_6.

[5] E. Bonabeau, M. Dorigo, and G. Theraulaz.1999 Swarm Intelligence: From Natural to Artifcial Systems. Oxford, U.K.: Oxford Univ. Press

[6] R. Poli, J. Kennedy, and T. Blackwell. 2010.Particle swarm optimization. Swarm Intell., vol. 1, no. 1, pp. 33_57, doi: https://doi.org/10.1007/s11721-007-00020 .
[7] M. Dorigo.1992.Optimization, learning and natural algorithms. Ph.D. dissertation, Politecnico di Milano, Milan, Italy.

[8] C. S. Moreau, C. D. Bell, R. Vila, S. B. Archibald, and N. E. Pierce 2006.Phylogeny of the ants: Diversi_cation in the age of angiosperms. Science,vol. 312, no. 5770, pp. 101_104.

[9] G. F. Oster and E. O. Wilson.1978 Caste and Ecology in the Social Insects. Princeton, NJ, USA: Princeton Univ. Press.

[10] T. Flannery. 2011.Here on Earth: A Natural History of the Planet. New York, NY, USA: Grove.

[11] H. Zhang, X. Wang, P. Memarmoshrefi and D. Hogrefe.2017.A Survey of Ant Colony Optimization Based Routing Protocols for Mobile Ad Hoc Networks. In IEEE Access, vol. 5, pp. 24139-24161.

[12] F.Moyson and B. Manderick.1988. The Collective Behavior of Ants: An Example of Self-organization in Massive Parallelism, Vrije Univ. Brussel, Ixelles, Belgium.

[13] S. Goss, S. Aron, J.-L. Deneubourg, and J. M. Pasteels.1989.Self-organized shortcuts in the Argentine ant. Naturwissenschaften, vol. 76, no. 12,pp. 579581

[14] M. Dorigo and T. Stützle.2004.Ant Colony Optimization. Cambridge, MA, USA: MIT Press.

[15] K. Anuj Gupta, K. Anil. Verma, and H. Sadawarti.2011.Analysis of various Swarm-based and Ant-based Algorithms. In Proc. Of International Conference on Advances in Computing and Artificial Intelligence (ACAI 2011), an ACM Chapter Event, Chitkara University, Punjab, 21-22 , pp - 39-43.

[16] G. Di Caro and M. Dorigo.1998.AntNet: Distributed stigmergetic control for communications networks," J. Artif. Intell. Res., vol. 9, pp. 317_365.

[17] M. Gunes, U. Sorges, and I. Bouazizi.2002.ARA-the ant-colony based routing algorithm for MANETs," in Proc. Int. Conf. Parallel Process. Workshops, pp. $79 \_85$.

[18] J. S. Baras and H. Mehta.2003.A probabilistic emergent routing algorithm for mobile ad hoc networks. In Proc. Modeling Optim. Mobile, Ad Hoc Wireless Netw. (WiOpt), p. 10.

[19] C. Perkins, E. Belding-Royer, and S. Das.2003.Ad hoc on-demand distance vector (AODV) routing. IETF, Fremont, CA, USA,Tech. Rep. rfc3561. Accessed: Oct. 26, 2017.2 [Online]. Available:https://tools.ietf.org/html/rfc3561

[20] G. Di Caro, F. Ducatelle, and L. M Gambardella.2005.AntHocNet: An adaptive natureinspired algorithm for routing in mobile ad hoc networks. Eur.Trans. Telecommun., vol. 16, no. 5, pp. 443455

[21] S. K. Nivetha, R. Asokan and N Senthilkumaran.2013.A swarm-based hybrid routing protocol to support multiple Quality of Service (QoS) metrics in mobile ad hoc networks, Fourth International Conference on Computing, 
International Journal of Computer Applications (0975 - 8887)

Volume 181 - No. 35, January 2019

Communications and Networking Technologies (ICCCNT), Tiruchengode, pp. 1-8

[22] E. Osagie, P. Thulasiraman, and R. K. Thulasiram.2008. PACONET: Improved ant colony optimization routing algorithm for mobile ad hoc networks. In Proc. 22nd Int. Conf. Adv. Inf. Netw. Appl. (AINA), pp. 204_211
[23] J. Wang, E. Osagie, P. Thulasiraman, and R. K. Thulasiram.2009. HOPNET: A hybrid ant colony optimization routing algorithm for mobile ad hoc network," Ad Hoc Netw., vol. 7, no. 4, pp. 690_705. 